\begin{tabular}{|c|l|}
\hline Title & Characterization of internal fracture process of double network hydrogels under uniaxial elongation \\
\hline Author(s) & Nakajima, Tasuku; Kurokawa, Takayuki; A hmed, Saika; Wu, Wen-li; Gong, Jian Ping \\
\hline Citation & $\begin{array}{l}\text { Soft Matter, 9(6), 1955-1966 } \\
\text { https:/doi.org/10.1039/62sm27232f }\end{array}$ \\
\hline Issue Date & 2013-02-14 \\
\hline Doc URL & http://hdl.handle.net/2115/53694 \\
\hline Rights & Soft Matter, 2013,9, 1955-1966- Reproduced by permission of The Royal Society of Chemistry (RSC) \\
\hline Type & article (author version) \\
\hline File Information & SM9-6_1955-1966.pdf \\
\hline
\end{tabular}

Instructions for use 


\title{
Characterization of Internal Fracture Process of Double Network Hydrogels under Uniaxial Elongation
}

\author{
Tasuku Nakajima, ${ }^{a}$ Takayuki Kurokawa, ${ }^{a, b}$ Saika Ahmed, ${ }^{c}$ Wen-li Wu ${ }^{d}$ and Jian Ping Gong*a \\ Received (in $X X X, X X X)$ Xth $X X X X X X X X X 20 X X$, Accepted Xth XXXXXXXXX 20XX \\ ${ }_{5}$ DOI: 10.1039/b000000x
}

Previously we revealed that the high toughness of double network hydrogels (DN gels) derives from the internal fracture of the brittle network during deformation, which dissipates energy as sacrificial bonds. In this study, we intend to elucidate the detailed internal fracture process of DN gels. We quantitatively analysed the tensile hysteresis and re-swelling behaviour of a DN gel that shows a well-defined necking 10 and strain hardening, and obtained the following new findings: 1) Fracture of the $1^{\text {st }}$ network PAMPS starts far below the yielding strain, and $90 \%$ of the initially load-bearing PAMPS chains already breaks at the necking point. 2) The dominant internal fracture process occurs in the necking and hardening region although the softening mainly occurs before necking. 3) The internal fracture efficiency is very high, $85 \%$ of the work is used for the internal fracture and $9 \%$ of all PAMPS chains break at sample failure. 4) The

15 internal fracture is anisotropic, fracture occurs preferentially perpendicular to the tensile direction than other two directions, but the fracture anisotropy decreases in the hardening region. Result 1) and 2) is in agreement with a hierarchical structural model of PAMPS network. Based on these findings, we present a revised description of the fracture process of DN gels.

\section{Introduction}

20 Toughness is the most important parameter for characterizing the durability of materials. Tough materials such as metals and some plastics are difficult to break even when subject to enormously large impact, while strong but not tough materials such as glass cannot withstand impact. High toughness of these

25 materials is often resulted from an extensive structural transition during the deformation process. As it consumes a large amount of energy, the total work required for the fracture of such tough materials dramatically increases. Examples of structural transition are migration of the dislocation in metal $^{1}$, reorientation of the 30 crystalline structure in polyethylene ${ }^{2}$, and crazing of poly(methyl methacrylate $)^{3}$. These structural transitions can be detected typically as local plastic deformations (yielding) and large mechanical hysteresis.

Classic polymer gels, which contain a large amount of solvents 35 within their $3 \mathrm{D}$ polymer networks, are weak and brittle materials, with a fracture energy typically in the range of $1 \sim 10 \mathrm{~J} / \mathrm{m}^{24}$. The weakness (low strength) of the gels originates from their diluted and heterogeneous polymer density, while the intrinsic reason of the brittleness (low toughness) attributes to their purely elastic 40 nature, which implies absence of structural transition. In contrast, several kinds of newly developed robust gels show higher toughness than conventional gels ${ }^{5}$. Especially, the double network gels (DN gels), though with a high water content $(>90$ wt $\%$ ), have extremely high fracture energy of $\sim 10^{3} \mathrm{~J} / \mathrm{m}^{2}$. This 45 value is much higher than that of classic gels, and even comparable with that of some industrial rubbers ${ }^{6-8}$. The tough DN gels have a contrast structure that the second polymer network, flexible and loosely cross-linked, is densely packed and interpenetrated in the cage of first polymer network that is a rigid 50 skeleton, highly cross-linked and in dilute concentration. Such a structure is synthesized by a two-step network formation process using the polyelectrolyte poly(2-acrylamido-2-methylpropanesulfonic acid) gel (PAMPS gel) as the first network and the neutral polyacrylamide (PAAm) as the second network. This 55 contrast structure makes mechanical properties of DN gels significantly different from those of conventional interpenetrating networks (IPN) gels that do not possess such contrast structure.

DN gels also show a structural transition during deformation like other tough materials. Some DN gels show a yielding-like 60 phenomenon associated with the formation of a necking zone and irreversible softening (the Mullins effect) in fracture process ${ }^{9-11}$. These phenomena suggest some sort of structural fracture within DN gels. As these phenomena are almost rate-independent, effect of physical interaction such as solvent migration is negligible. ${ }_{65}$ Furthermore, in contrast to typical tough materials that show mainly plastic deformation beyond the yielding point, DN gels show negligible $(\sim 10 \%)$ residual strain and purely elastic deformation even after a large elongation. Based on these findings, we have proposed a picture where the first brittle 70 PAMPS network shows an "internal fracture", which dissipates energy as sacrificial bonds 9,10 . Figure 1 shows schematic illustrations of the suggested internal fracture process of DN gels. The first PAMPS network is rigid and brittle whereas the second PAAm network is soft and flexible. Thus, with deformation of the 75 flexible PAAm, the brittle PAMPS network breaks into small 


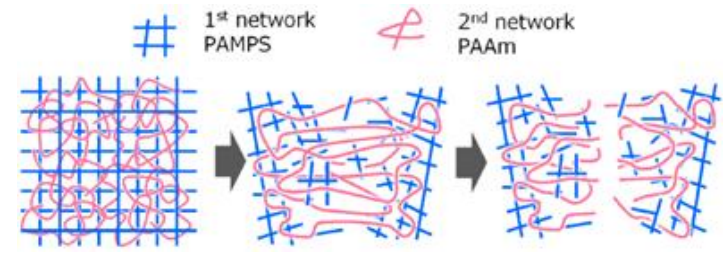

Figure 1 Previously proposed fracture model of Double Network gels, which roughly explains the internal fracture process of the brittle network $^{9,10}$. First, a large portion of the brittle PAMPS network (blue) breaks into fragments with elongation of the soft PAAm network (red).

5 Then, the fully stretched PAAm network breaks, which leads to final grand failure of the sample. As a large amount of energy is dissipated during the fracture of the brittle network, a large amount of energy is required for the crack propagation; in other words, DN gels show high toughness. Thus, the brittle network serves as "sacrificial bonds"8.

10 fragments. As a large quantity of energy is dissipated during this process, a large amount of energy is required for the crack propagation; in other words, DN gels show high toughness. This picture is supported by a number of experiments, such as partial change of the elastic modulus near a crack and surface 15 morphology upon deformation of DN gels ${ }^{9-11}$. Several theoretical models have been proposed based on this picture, which gives more insight of this interesting system ${ }^{12-14}$.

However, beside the above rough "internal fracture" picture, the detailed internal fracture process is still unknown; in 20 particular, exactly when and how the PAMPS network breaks, the amount of the covalent bonds that fractures, the characteristic size and shape of the fractured PAMPS networks, etc. Previously, the relationship between the amount of fractured PAMPS and the deformation strain has been quantitatively discussed based on the 25 loading-unloading hysteresis $U_{\text {hys }}$, which is the energy used for internal fracture. ${ }^{10}$ However, the measurements were performed under small deformations for the samples that did not show a distinct yielding. As the toughest DN gels shows a well-defined yielding and strain hardening, which are common characteristic 30 features for tough materials, in this study, we concentrated on the DN gels that show yielding (necking) and strain hardening, and we performed the following two experiments to gain further understanding of the internal fracture process of tough DN gels. The first experiment was a mechanical hysteresis measurement, 35 which is the easiest way to elucidate the internal fracture behaviour quantitatively. By measuring the loading-unloading hysteresis $U_{\text {hys }}$ and the decrease in the initial elastic modulus $E$, ratios of the fractured PAMPS networks were calculated quantitatively. The second experiment was a re-swelling 40 measurement. Once a DN gel experiences a large deformation, it gains extra-swelling in water due to the fracture of the PAMPS network. In general, swelling ratio of gels is determined as the balance between the osmotic pressure $\Pi$ of polymers and counter ions in the gels, and the elastic stress of the networks ${ }^{15}$. The 45 former term promotes swelling while the latter term suppresses it. In the case of the PAMPS gels in equilibrium swelling state, the network chains hold large elastic stress and are in fully-stretched state due to large $\Pi$ of counter ions. If the PAAm gels are synthesized inside the PAMPS gels to form DN structure, extra $\Pi$ 50 originated from dense PAAm network promotes further swelling of DN gels. However, the fully-stretched PAMPS network works as a cage and suppresses the swelling of the gels. When an internal fracture of the PAMPS network occurs in DN gels, the effect of swelling suppression decreases, and as a result, the (re55 )swelling ratio of DN gels increases. Therefore, the increase in the (re-)swelling ratio corresponds to an increase in the degree of fracture of the PAMPS network. We measured the variation in weight and size ( $x, y$, and $z$ direction) of $\mathrm{DN}$ gels after deformation and re-swelling in order to elucidate the extent of ${ }_{60}$ fracture of the PAMPS network and the internal fracture anisotropy.

Through these experiments, we found following results in this paper: A. Internal fracture starts before yielding, and continues until global fracture of the sample. B. The internal fracture 65 mainly occurs in the yielding and hardening region, with a high efficiency compared to rubber systems. C. DN gels show twostep internal fracture, which is in agreement with a hierarchical structural model. D. Early stage of fracture behaviour can be well-fitted with some theoretical models. E. Internal fracture of ${ }_{70} \mathrm{DN}$ gels is anisotropic. Based on these findings, we present a more detailed fracture process of highly deformable DN gels under uniaxial elongation.

\section{Experimental}

\section{Materials}

75 2-Acrylamido-2-methylpropanesulfonic acid (AMPS, Toa Gosei Co., Ltd.) was recrystallized from methanol. Acrylamide (AAm, Junsei Chemical Co., Ltd.) was recrystallized from chloroform. $N, N$ '-Methylenebisacrylamide (MBAA, Wako Pure Chemical Industries, Ltd.) was recrystallized from ethanol. 2${ }_{80}$ Oxoglutaric acid (Wako Pure Chemical Industries, Ltd.) was used as received.

\section{Synthesis of $t$-DN gels}

Truly-independent DN gels, or simply $t$-DN gels, are special types of DN gels that do not contain any covalent bonds between 85 the two networks ${ }^{7}$. As $t$-DN gels have a simpler structure than conventional DN gels, $t$-DN gels were useful for analysing the fracture process of DN gels. Firstly, the AMPS pre-gel aqueous solution containing $1 \mathrm{M}$ of AMPS, $4 \mathrm{~mol} \%$ of MBAA as the cross-linker, and $0.1 \mathrm{~mol} \%$ of 2-oxoglutaric acid as the photo90 initiator (the molar percentages are given with respect to the monomer) was prepared. The solution was then moved to an argon blanket and poured into a glass mould consisting of two flat glass plates separated by silicone rubber as a spacer (the thicknesses of glass plates and of a spacer were $3 \mathrm{~mm}$ and $1 \mathrm{~mm}$, 95 respectively). Photo-polymerization of PAMPS gels was performed by $365 \mathrm{~nm}$ UV irradiation for $8 \mathrm{~h}$. The PAMPS gels were then immersed in a $0.1 \mathrm{M}$ 2-oxoglutaric acid aqueous solution for $1 \mathrm{~d}$ and $\mathrm{UV}$ irradiated at $365 \mathrm{~nm}$ for $10 \mathrm{~h}$. In this process, excess radicals were generated in the gels that consumed 100 all the unreacted vinyl groups in the gels, making them inert. The gels were then soaked in pure water for $7 \mathrm{~d}$ in order to remove the excess photo-initiator. During this period, the water was replaced daily. The PAMPS gels were then immersed in an AAm pre-gel aqueous solution consisting of $2 \mathrm{M}$ of AAm, $0.02 \mathrm{~mol} \%$ of 105 MBAA, and $0.01 \mathrm{~mol} \%$ of 2-oxoglutaric acid for at least $2 \mathrm{~d}$. The solutions were replaced once during immersion. After immersion, the gels were sandwiched between two flat glass plates under argon atmosphere. Photo-polymerization of PAAm gels was performed in the presence of the PAMPS gels by UV irradiation 


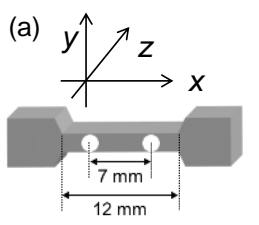

(b)

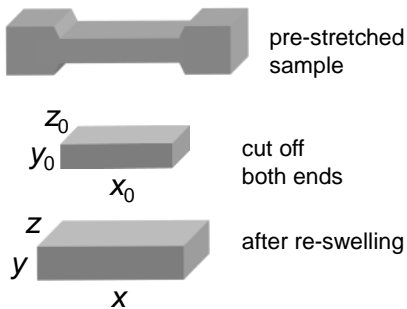

Figure 2 (a) A sample for uniaxial tensile test. Two white spots are dotted with a common white pen to calculate accurate strain. (b) Samples for reswelling test.

at $365 \mathrm{~nm}$ for $9 \mathrm{~h}$. These $t$-DN gels were then soaked in pure 5 water for at least $5 \mathrm{~d}$ in order to remove any un-reacted reagents.

\section{Tensile Test and Mechanical Hysteresis Measurements}

Uniaxial tensile test was carried out on the dumbbell-shaped samples standardized to the JIS-K6251-7 size (12 mm in $x$-axis (length), $2 \mathrm{~mm}$ in $y$-axis (width), and $2.4 \mathrm{~mm}$ in $z$-axis 10 (thickness)) with a commercial tensile tester Instron 5965 (Instron Co.) with a non-contacting video extensometer AVE (Instron Co.). Two white spots with distance of about $7 \mathrm{~mm}$ were dotted on the samples with a common white pen as shown in Figure 2(a), and the samples were stretched along $x$-axis at constant velocity 15 of $100 \mathrm{~mm} / \mathrm{min}$ determined according to JIS-K6251. The accurate strain of $x$-axis $\varepsilon$ was measured by real-time observation of distance between the two white spots with the video extensometer The initial elastic modulus $E_{\mathrm{DN}}(\mathrm{MPa})$ was determined from the best linear fitting of stress-strain curves using the relationship $20 \sigma=E_{\mathrm{DN}} \times \varepsilon$ at a strain of $\varepsilon<10 \%$, as Hooke's law can be applied approximately to rubberlike materials (including gels) if strain is close to 0 .

Tensile hysteresis measurements were carried out on the same specimens. The samples were first elongated to a certain strain $\varepsilon_{1}$ $25(\mathrm{~m} / \mathrm{m})$ and then immediately unloaded (the unloading process was not recorded). Then, after recovering their original length, they were elongated immediately to the strain $\varepsilon_{2}\left(\varepsilon_{2}>\varepsilon_{1}\right)$ and unloaded again. The measurement was similarly carried out at $\varepsilon_{3}, \varepsilon_{4}, \ldots \varepsilon_{\mathrm{n}}$ until their breaking point. In general, the hysteresis measurement 30 is carried out by measuring the (first) loading and unloading curves. However, this time measurement of unloading curves was impossible due to limitation of the equipment. In the case of DN gels, the second loading curve overlaps the first unloading curve almost completely even at large strain (refer Figure S1 of 35 Supplemental Information). Thus, in this study the $(n+1)$ th loading curve was measured instead of the $n$th unloading curve. The initial elastic modulus $E_{\mathrm{DN}}(\mathrm{MPa})$ after deformation as a function of $\varepsilon_{\max }$ (the maximum $\varepsilon$ the sample experienced) was calculated from the loading curves, as explained above. The work 40 of extension $W\left(\mathrm{MJ} / \mathrm{m}^{3}\right)$ as a function of $\varepsilon_{\max }$ was defined as the area under the tensile stress-strain curves. The dissipated energy, $U_{\text {hys }}\left(\mathrm{MJ} / \mathrm{m}^{3}\right)$, which is the area between two successive loading curves, was calculated as a function of $\varepsilon_{\max }$ as

$$
\begin{aligned}
U_{h y s_{-} 1} & =\int_{0}^{\varepsilon 1} \sigma_{1 s t} d \varepsilon-\int_{0}^{\varepsilon 1} \sigma_{2 n d} d \varepsilon \\
{ }_{45} U_{h y s_{\_} n} & =U_{h y s_{-} n-1}+\int_{0}^{\varepsilon n} \sigma_{n t h} d \varepsilon-\int_{0}^{\varepsilon n} \sigma_{n+1 t h} d \varepsilon
\end{aligned}
$$

where $U_{\text {hys_n }}$ denotes the total accumulated dissipated energy at the strain $\varepsilon_{\mathrm{n}}\left(=\varepsilon_{\mathrm{max}}\right)$.

\section{(Re-)Swelling Ratio and Swelling Anisotropy Measurements}

The dumbbell-shaped DN gels were pre-stretched along $x$-axis 50 to a certain strain $\varepsilon_{\max }$ with constant velocity of $100 \mathrm{~mm} / \mathrm{min}$, and unloaded (relaxed). The relaxed DN gels showed $\sim 10 \%$ of residual strain, slightly depending on the $\varepsilon_{\max }$. As the value of residual strain does not depend on time, it might be originated from structural change of the DN gels, caused by internal fracture 55 of the PAMPS network. The samples in this state (pre-stretched sample) were used as reference state for determination of reswelling ratio. Both ends of the pre-stretched DN samples were cut off, and the reference lengths of centre part of the samples, $x_{0}$, $y_{0}$, and $z_{0}$, were measured using a caliper as shown in Figure 2(b). ${ }_{60}$ The samples were then immersed in pure water for $5 \mathrm{~d}$, and after re-swelling, the lengths in each direction, $x, y$, and $z$, were measured using a caliper. The swelling ratios of $x, y$, and $z$-axis are expressed as $\alpha_{\mathrm{x}}, \alpha_{\mathrm{y}}$, and $\alpha_{\mathrm{z}}$, respectively, where $\alpha_{i}=i / i_{0}(i=x$, $y$, or $z$ ). The swelling anisotropy, $Q$, is defined as

${ }_{65} Q=\frac{2 \alpha_{\mathrm{x}}}{\alpha_{\mathrm{y}}+\alpha_{\mathrm{z}}}$

For partly necked samples, these values were not measured due to heterogeneous swelling.

The (re-)swelling degree, $q_{\mathrm{w}}$, was also measured independently. The degree $q_{\mathrm{w}}$ is defined based on the weight as $q_{\mathrm{w}}(\mathrm{g} / \mathrm{g})=$ ${ }_{70} W_{\text {swell }} / W_{\text {dry }}$, where $W_{\text {swell }}$ denotes the weight of a re-swollen gel and $W_{\text {dry }}$ denotes that of a dried gel. $W_{\text {swell }}$ and $W_{\text {dry }}$ were measured using a moisture balance MOC-120H (Shimadzu Co.). The heating temperature for drying was $120^{\circ} \mathrm{C}$.

\section{Results and Discussion}

\section{Mechanical Hysteresis}

Figure 3(a) shows the nominal stress-strain curve (s-s curve) of the synthesized $t$-DN gels with high toughness. Shape of the s-s curve is similar to that of previously-reported tough DN gels ${ }^{9,16}$. The s-s curve can be divided into three regions. The first region, 80 before the yielding point, is called the pre-necking region. In this region, deformation was homogenous with no strain localization. After yielding, a portion of the sample constricted as a neck (necking zone), and the stress became constant and independent of the strain while the narrow necking zone developed with the 85 increase of strain. Such a plateau region in the s-s curve is called the necking region. After the necking zone reached the end of the sample, the sample regained a homogenous deformation. This final region is called the hardening region because previous reports imply that strain-hardening of the PAAm chains may 90 occur in this region ${ }^{9,11}$. Illustrations of deformed DN gels in each region are shown in Figure 3(b). 
(a)
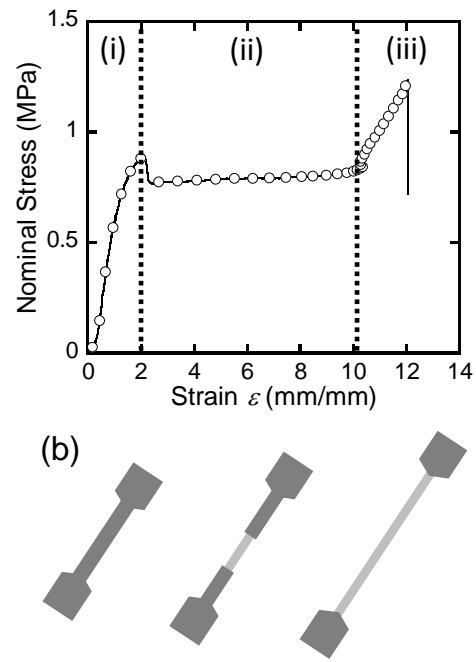

(i) Pre-Necking (ii) Necking (iii) Hardening

Figure 3 (a) The stress-strain curve of the $t$-DN gel measured by the tensile test. The tensile velocity was fixed at $100 \mathrm{~mm} / \mathrm{min}$. For clarity, only some of the data are displayed in the graph. The curve can be divided into three regions as shown in the figure, namely (i) pre-necking,

5 (ii) necking, and (iii) hardening regions. (b) Illustrations of deformed DN gels in these three regions. Dark grey and light grey regions represent unyielding and yielding zones, respectively.

(a)

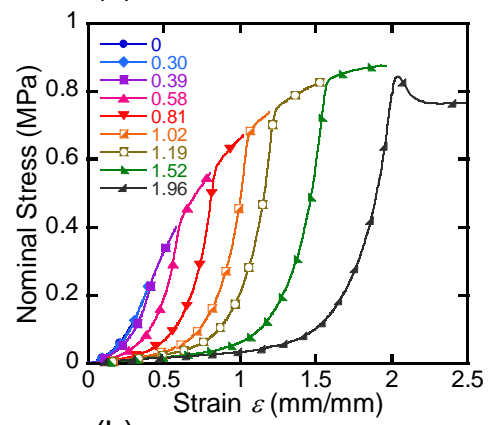

(b)

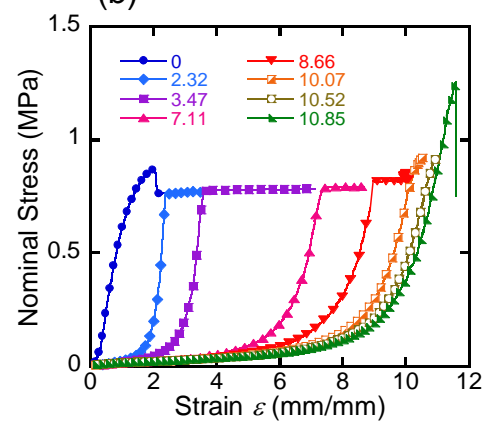

Figure 4 Tensile hysteresis loops of $t$-DN gels in (a) the pre-necking 10 region and (b) the necking/hardening regions measured in a cyclic tensile test. For clarity, only some of the data are displayed in the graphs. The symbol numbers denote the pre-experienced strain, $\varepsilon_{\max }$, before measurement of each stress-strain curve. The tensile velocity was fixed at $100 \mathrm{~mm} / \mathrm{min}$.
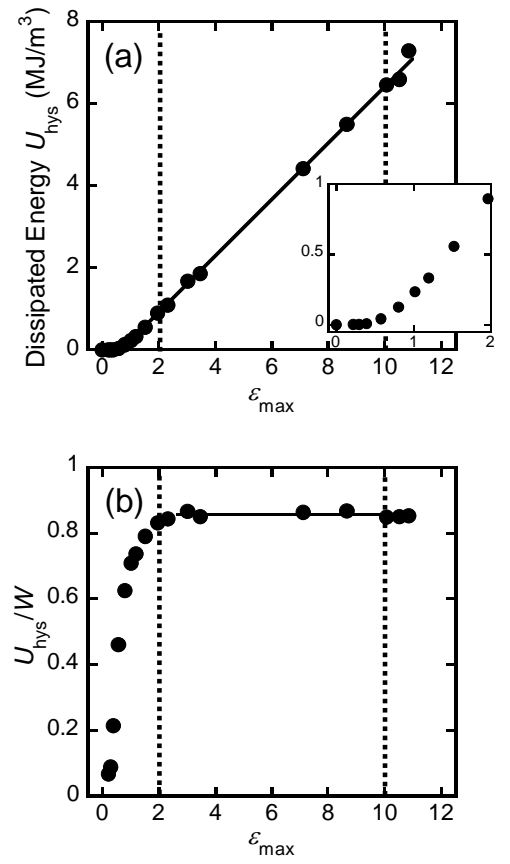

15 Figure 5 (a) Dissipated energy, $U_{\text {hys }}$, dependence on $\varepsilon_{\max }$ of $t$-DN gels. The small graph is an enlarged figure in the pre-necking region. (b) $U_{\text {hys }} / W$ (ratio of the irreversible work within the total work done by extension) dependence on $\varepsilon_{\max }$ of $t$-DN gels. The solid lines are guides for the eye. Two dotted vertical lines indicate the boundary of the three 20 regions described in Figure 3(a).

Figure 4 shows the tensile mechanical hysteresis loops of the $t$ DN gels. The significantly large hysteresis, implying PAMPS internal fracture, was observed in all the three regions. It is notable that the mechanical hysteresis first appeared when the ${ }_{25}$ pre-experienced strain $\varepsilon_{\max }$ reached approximately $40 \%$. These facts mean that the internal fracture of PAMPS started at far below the yielding point, and continued during the elongation process. Surprisingly, the critical strain for mechanical hysteresis of $40 \%$ is very close to the fracture strain, $\varepsilon_{\mathrm{f}}$, of the swollen ${ }_{30}$ PAMPS single network gels (about $40 \%$ ). ${ }^{17}$ The same value of $40 \%$ was also observed in $t$-DN gels with different PAAm concentrations (data not shown). The consistency of these values of 0.4 implies that the onset of the crack growth is the same for PAMPS single network and PAMPS network in the DN gels. In 35 the case of single network gels, an initiated crack catastrophically propagates and induces total sample breakage. In contrast, in the case of DN gels such catastrophic crack propagation is substantially suppressed by the presence of the second PAAm network, although the PAAm network does not prevent crack 40 initiation of the PAMPS network.

Figure 5(a) shows the dependence of the amount of dissipated energy $U_{\text {hys }}$ on $\varepsilon_{\max }$ of the $t$-DN gels. When $\varepsilon_{\max }$ was smaller than the critical value of $40 \%, U_{\text {hys }}$ was almost equal to zero, as the PAMPS network in the DN gels did not break. When $\varepsilon_{\max }$ became ${ }_{45}$ larger than $40 \%, U_{\text {hys }}$ gradually increased with $\varepsilon_{\max }$. After reaching the yielding point, $U_{\text {hys }}$ linearly increased with $\varepsilon_{\max }$. The slope $d U_{\text {hys }} / d \varepsilon_{\max }$ became constant over the necking and hardening regions, implying the internal fracture continued with deformation until reaching the global fracture point. It means that, ${ }_{50}$ in hardening region, not only PAAm strain hardening but also PAMPS internal fracture occurs continuously. 


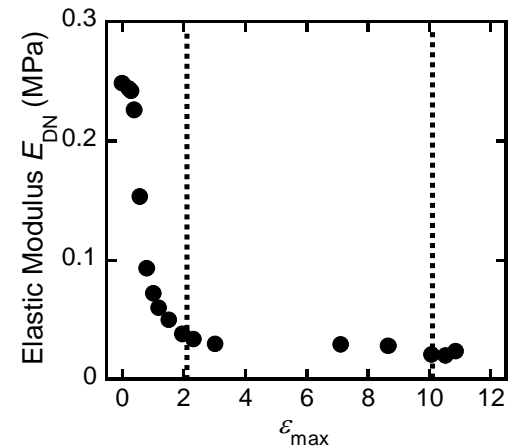

Figure 6 Initial elastic modulus, $E_{\mathrm{DN}}$, of $t$-DN gels after pre-stretched to various strains, $\varepsilon_{\max }$. It should be noted that $E_{\mathrm{DN}}$ was determined from the initial slope of the second loading curves of the pre-stretched DN gels.

Two dotted vertical lines indicate the boundary of the three regions described in Figure 3a.

One of the features of the DN gels is that efficiency of energy dissipation is larger than that of vulcanized rubbers, which are other examples of tough yet soft materials. In order to illustrate this fact qualitatively, the relationship between $U_{\text {hys }} / W$ and $\varepsilon_{\max }$ is 10 shown in Figure 5(b), where $W$ is the work done by extension and the ratio $U_{\text {hys }} / W$ represents the fraction of the irreversible work (due to the internal fracture of the PAMPS network) within the total work of extension. After reaching the yielding point, $U_{\text {hys }} / W$ showed a constant value of approximately $85 \%$, regardless the 15 increase in the strain. This result implies that $85 \%$ of the work was used for the internal fracture of the PAMPS network. The value of $U_{\text {hys }} / W$ of the DN gels is higher than that of vulcanized rubbers $(59-67 \%)^{18}$. Although several theories have been proposed for the softening (Mullins effect) of rubbers, there is no 20 doubt that their mechanical hysteresis is derived from a large structural transition. Typically, polymer chains in rubbers are in a randomly coiled (relaxed) state. Therefore, a large deformation is required to induce a structural transition in rubbers. In contrast, chains in the first network of DN gels are in a fully-stretched state 25 due to the nature of the strong polyelectrolyte. Therefore, a small deformation is enough to induce a structural transition (internal fracture). Thus, the ratio $U_{\text {hys }} / W$ of $\mathrm{DN}$ gels is higher than that of rubbers.

\section{Initial Elastic Modulus Change}

30 Figure 6 shows the initial elastic modulus $E_{\mathrm{DN}}$ calculated from the second loading curves of the $t$-DN gels after experienced various strains $\varepsilon_{\max }$. According to the classical rubber elasticity theory, $E_{\mathrm{DN}}$ is proportional to the number density of "elastically effective" chains, which are the chains that actually hold the 35 stress when the bulk gels are slightly deformed ${ }^{19}$. In the prenecking region, with the increase of $\varepsilon_{\max }, E_{\mathrm{DN}}$ dramatically decreased from $0.25 \mathrm{MPa}$ (virgin state) to $0.038 \mathrm{MPa}$ (end of the pre-necking region), a difference of about one order of magnitude. The remarkable decrease in $E_{\mathrm{DN}}$ indicates that most of the 40 elastically effective PAMPS chains of the bulk gel were already broken in this region. In the necking region, a slight decrease of $E_{\mathrm{DN}}$ (from $0.038 \mathrm{MPa}$ to $0.020 \mathrm{MPa}$ ) was observed, which is due to the growth of necked zone at the expense of the remaining unnecked zone, as the necked zone has a lower modulus than that of 45 the un-necked zone ${ }^{9}$. In the hardening region, the value of $E_{\mathrm{DN}}$ (about $0.02 \mathrm{MPa}$ ) remained almost constant with increasing $\varepsilon_{\max }$ and was close to that of the single PAAm gels $(0.018 \mathrm{MPa})^{9}$. This indicates that the further fracture of PAMPS into smaller size does not influence the overall stiffness of the gel in this region. 50 These results demonstrate that the bulk continuous PAMPS network starts to break into discontinuous fragments in the necking region, and after reaching the hardening region, these PAMPS fragments further break into smaller fragments with increase in $\varepsilon$. It should be noted that these results on the fracture ${ }_{55}$ behaviours were not notified in our previous studies ${ }^{9}$. In those studies, we had assumed that before yielding, the fracture of PAMPS and the modulus decrease are negligible, whilst in the yielding region, the PAMPS network fractures into discontinuous clusters, leading to an abrupt decrease in the modulus. The co${ }_{60}$ existence of the yielded zone and the un-yielded zone consists of a softened (discontinuous) and an un-softened (continuous) PAMPS network.

\section{Estimation of the fractions of fractured PAMPS}

Here, in order to discuss the internal fracture behaviour ${ }_{65}$ quantitatively, we quantify that how much amount of PAMPS network fractures due to deformation. Two fractions of fractured PAMPS were calculated based on the different experimental results. One is the fraction of the fractured PAMPS chains out of the "elastically effective" PAMPS chains $\phi_{\mathrm{m}}$, which can be 70 calculated from the variation in the modulus $E_{D N}$ of the samples. The second fraction is the fractured PAMPS chains out of "all" nominally PAMPS chains, $\phi_{b}$, which can be calculated from the dissipated energy, $U_{\text {hys }}$ and the PAMPS concentration.

The $\phi_{m}$ is defined as

${ }_{75} \phi_{m}=1-\frac{v_{e}}{v_{e 0}}$

where $v_{\mathrm{e}}$ and $v_{\mathrm{e} 0}\left(1 / \mathrm{m}^{3}\right)$ denote the number density of elastically effective PAMPS chains in the elongated and virgin DN gels, respectively. Since the modulus of a polyelectrolyte gel is the product of the energy for stretching a chain and the number 80 density of chains, ${ }^{19}$ the ratio $v_{\mathrm{e}} / v_{\mathrm{e} 0}$ can be calculated by

$v_{e} / v_{e 0}=E_{P A M P S} / E_{P A M P S O}$

where $E_{\mathrm{PAMPS}}$ and $E_{\mathrm{PAMPS} 0}$ are the initial elastic moduli of the PAMPS component of pre-streched and virgin DN gels, respectively. Thus, $\phi_{\mathrm{m}}$ can be calculated as

${ }_{85} \phi_{m}=1-\frac{E_{P A M P S}}{E_{P A M P S 0}}$

As $E_{\text {PAMPS }}$ cannot be measured directly due to complex structure of DN gels, we estimated $E_{\mathrm{PAMPS}}$ from the initial elastic modulus of DN gels, $E_{\mathrm{DN}}$. We assumed that the modulus of DN gels is the sum of the moduli of the two networks:

${ }_{90} E_{D N}=E_{P A M P S}+E_{P A A m}$

As PAAm gels do not show mechanical hysteresis ${ }^{20}$, the initial elastic modulus of the PAAm component, $E_{\text {PAAm }}$, should be constant during the mechanical hysteresis measurements. Although $E_{\text {PAAm }}$ cannot be measured accurately, it is assumed to 95 be close to the value of single PAAm network gels. As the modulus of the single PAAm gel was found to be $0.018 \mathrm{MPa}$ for this system ${ }^{9}$, finally we obtain 
(a)

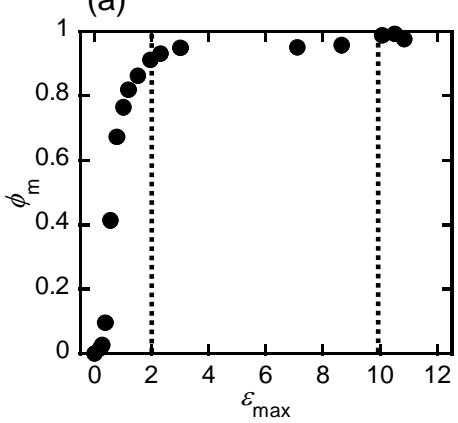

(b)

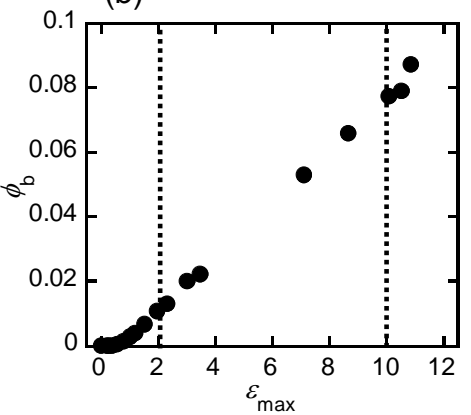

(c)

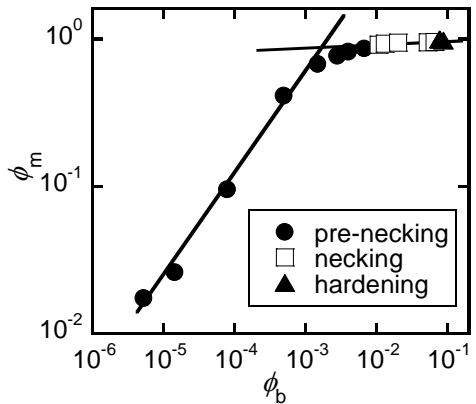

Figure $7(\mathrm{a}, \mathrm{b})$ Fraction of fractured PAMPS chains in $t$-DN gels, $\phi$ estimated based on (a) a decrease of the modulus ( $\left.\phi_{\mathrm{m}}\right)$ and (b) the mechanical hysteresis loops $\left(\phi_{b}\right)$. Two dotted vertical lines indicate the boundary of the three regions described in Figure 3(a). (c) Relationship between $\phi_{\mathrm{m}}$ and $\phi_{b}$.

$\phi_{\mathrm{m}} \approx 1-\frac{E_{D N}-0.018}{E_{D N 0}-0.018}$

Figure 7(a) shows the relationship between the calculated $\phi_{\mathrm{m}}$ and ${ }_{5} \varepsilon_{\max }$. The fracture ratio, $\phi_{\mathrm{m}}$, increases significantly with increasing $\varepsilon_{\max }$ in the pre-necking region, and reaches $90 \%$ at the yielding point. After reaching the yielding point, $\phi_{\mathrm{m}}$ increases slightly and reaches almost $100 \%$ at the end of the necking region. This indicates that $90 \%$ and almost $100 \%$ of the elastically effective 10 chains of PAMPS have fractured at the yielding point and at the end of necking, respectively. The latter means that the PAMPS network has been fractured into fragments with percolated microcracks.

The $\phi_{b}$ is defined based on the dissipated energy. As the DN 15 gels showed completely irreversible hysteresis, we assumed that all the dissipated energy $U_{\text {hys }}$ was used for internal fracture of the PAMPS network. Considering this assumption, the $\phi_{b}$ can be expressed as

$\phi_{b}=\frac{U_{\text {hys }}}{U_{\text {total }}}$

20 where $U_{\text {hys }}(\varepsilon)\left(\mathrm{J} / \mathrm{m}^{3}\right)$ is the dissipated energy per unit volume at a pre-strain $\varepsilon_{\max }$ (the energy actually used for PAMPS internal fracture) and $U_{\text {total }}\left(\mathrm{J} / \mathrm{m}^{3}\right)$ is the energy theoretically required for the fracture of all the PAMPS chains within a unit volume. To consider $U_{\text {total }}$, we first estimated the energy required for breaking 25 one PAMPS chain, $U_{\text {single }}(\mathrm{J})$, by applying classical Lake-Thomas theory ${ }^{4}$ as

$U_{\text {single }}=N U_{\mathrm{b}}$

where $N$ is the number of C-C bonds in the main chain backbone of one chain and $U_{\mathrm{b}}(\mathrm{J})$ is the bond dissociation energy of one C-

${ }_{30} \mathrm{C}$ bond. eq.(10) means that the work to "rip" the polymer chain with $N$ bond is equal to the energy of disrupting all C-C bonds in the chain $N U_{\mathrm{b}}$ since these $\mathrm{C}-\mathrm{C}$ bonds are in series. If there are $n$ PAMPS chains in unit volume, $U_{\text {total }}$ can be calculated by

$U_{\text {total }}=\sum_{j=1}^{n} N_{j} U_{\mathrm{b}}$

35 where $N_{j}$ represents the number of C-C bonds in the $j^{\text {th }}$ PAMPS chain. It means that $U_{\text {total }}$ can be estimated by multiplying the number density of C-C bonds of PAMPS main chains with $U_{b}$. Thus, eq. (11) can be written a
$U_{\text {total }}=2 c_{1 s t} N_{A} U_{b}$

40 where $c_{1 \mathrm{st}}\left(\mathrm{mol} / \mathrm{m}^{3}\right)$ is the molar concentration of the PAMPS component and $N_{\mathrm{A}}$ is the Avogadro's constant $(1 / \mathrm{mol})$. The ensemble $2 c_{1 \mathrm{st}} N_{\mathrm{A}}$ represents the number of C-C bonds in the main chains per unit volume (the coefficient 2 accounts for the two $\mathrm{C}$ $\mathrm{C}$ bonds in each monomer unit in the main chains). This equation 45 implies that $U_{\text {total }}$ depends only on $c_{1 \mathrm{st}}$ and not directly on the structure of the PAMPS network. We used $N_{\mathrm{A}} U_{\mathrm{b}}=347(\mathrm{~kJ} / \mathrm{mol})$, which is the literature value for the $\mathrm{C}-\mathrm{C}$ bond dissociation energy in $\mathrm{C}_{2} \mathrm{H}_{5}-\mathrm{C}_{2} \mathrm{H}_{5}{ }^{21}$. Figure 7(b) shows the estimated $\phi_{b}$ of the $t$-DN gels. The resulting value of $\phi_{b}$ increases gradually with $\varepsilon_{\max }$, and 50 it finally reaches approximately $9 \%$ at the global fracture point. Here, two facts should be noticed. First, $9 \%$ of $\phi_{b}$ is very high. In the case of conventional gels, fracture of covalent bonds occurs only near the fractured surface, which means a vanishingly small $\phi_{b}$ (no or slight mechanical hysteresis). The contrasting double 55 network structure of DN gels induces an efficient PAMPS fracture even beneath the fractured surface, which leads to such high $\phi_{b}$. Second, the results for $\phi_{b}$ are rather different from those for $\phi_{\mathrm{m}}$. In all regions, $\phi_{\mathrm{m}}$ is much larger than $\phi_{b}$. The previous paper also reports similar results, though the measurement was ${ }_{60}$ performed on a sample that does not show necking ${ }^{10}$. To further demonstrate this result, the relationship between $\phi_{\mathrm{m}}$ and $\phi_{b}$ in log scale is shown as Figure 7(c). The data can be fitted by two power laws, which suggest existence of hierarchical internal fracture process of PAMPS network. When $\phi_{b}$ is below 0.001 65 (first process), $\phi_{\mathrm{m}} \sim \phi_{b}{ }^{0.7}$. The power of 0.7 (close to 1) means that increase of $\phi_{b}$ directly affects to $\phi_{\mathrm{m}}$. It indicates that the initially elastically effective polymer chains are mainly breaks in this process. In contrast, when $\phi_{b}$ is above 0.001 (second process), $\phi_{\mathrm{m}}$ is almost insensitive to the increase in $\phi_{b}$, indicating that the 70 discontinuous (initially elastically ineffective) PAMPS network mainly breaks into smaller fragments in this process. The first process approximately corresponds to pre-necking region, and the second process corresponds to necking and hardening regions. It should be noted that the former process requires only $0.1 \%$ of $\phi_{b}$,

75 which is only $1 / 100$ of $\phi_{b}$ at the sample failure. It means that the latter process, in which PAMPS fragments fracture into smaller pieces, is much more important for high toughness of DN gels.

The neutron scattering study has revealed a very heterogeneous structure of PAMPS hydrogel ${ }^{22}$. We discuss the 80 correlation between the hierarchical, two-step internal fracture 
and the heterogeneity of the PAMPS network. It is well known that common gels contain spatial inhomogeneity in the network $^{23,24}$. One reason is frozen concentration fluctuation by gelation $^{23}$. The other reason, which is specific for gels 5 synthesized from monomers and cross-linkers, is hierarchical gelation process which results in spatial inhomogeneity of crosslinking points ${ }^{24}$. Based on the scattering results, it is deduced that; in gelation process, 1) small gel clusters (microgels) are formed first, then 2) the microgels are connected to form bulk gels 10 containing large inhomogeneity, as illustrated in Figure $8(a, b)^{24}$. Assuming that the fracture of PAMPS does not occur catastrophically in the DN gel, the two-step internal fracture of PAMPS seems in agreement with this hierarchical structural model. According to this model, PAMPS chains can be classified 15 into two types. One is the chains constructing microgels. The other is the chains connecting microgels. The results of Figures 6 and 7 suggest that most of chains may belong to the former class and they do not contribute to the bulk modulus of the gel. Only a small amount of chains belong to the latter class and they are the 20 elastically effective chains contributing to the bulk modulus of PAMPS. According to this picture, we can illustrate initial PAMPS fracture behaviour as Figure $8(\mathrm{c}, \mathrm{d})$. As microgels consist of multiple polymer chains, the stress in microgel network is dispersed on many chains. In contrast, as microgels are 25 sparsely cross-linked by connecting chains, the stress in connecting chains is concentrated on few chains. Thus, at small strain (corresponding to pre-necking region), the connecting chains bear much larger stress and break preferentially. (a)

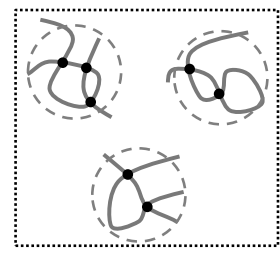

(c)

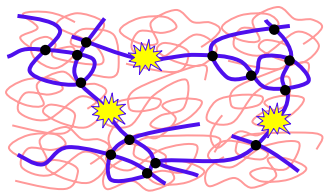

(b)

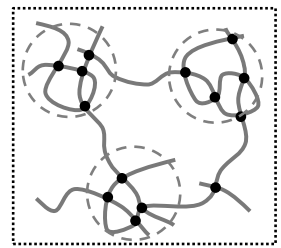

(d)

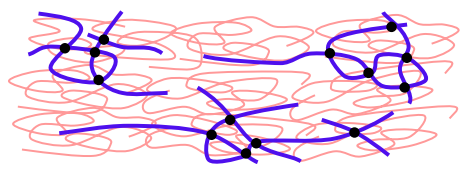

Figure 8 (a, b) Schematic illustrations of gelation process. Microgels are 30 formed first (a), and then they are connected to form bulk gel (b). Thus, the bulk gels contain large spatial inhomogeneity. (c, d) Schematic

illustrations of internal fracture process of DN gel at small strain (before necking) based on inhomogeneous structure model of PAMPS (blue). Stress concentrates on the PAMPS chains connecting microgels, and 35 these chains break first (c). After that, the load is borne by the PAAm chains (red) at small strain and PAMPS chains do not contribute to the initial elastic modulus $E_{\mathrm{DN}}(\mathrm{d})$. As a result, $E_{\mathrm{DN}}$ is insensitive to the further fracture of the PAMPS chains in the microgels in the large strain region (beyond necking), as observed in Figures 6 and 7.

40 After breakage of these chains, the PAMPS network becomes discontinuous and hardly bears stress at small strain, and the stress is borne by the PAAm chains. Thus, the PAMPS network no longer contributes to the initial elastic modulus $E_{\mathrm{DN}}$, although only $0.1 \%$ of the PAMPS chains break. At large strain 45 (corresponding to necking and hardening regions), however, stress is transferred from the PAAm network to the PAMPS microgels due to entanglement between two networks ${ }^{25,26}$. As a result, the PAMPS microgels further break into smaller fragments with strain. Thus, $\phi_{b}$ still increase until global fracture even if the ${ }_{50}$ PAMPS network is discontinuous. In contrast, $\phi_{\mathrm{m}}$ does not increase since all the initial stress bearing chains (the chains connecting the microgels) break at the necking region. This is the reason of large discrepancy of $\phi_{\mathrm{m}}$ and $\phi_{b}$. The heterogeneity effect of the PAMPS network on the fracture behaviour of DN gels has ${ }_{55}$ also been discussed by the previous work ${ }^{10}$. However, since the study was performed at a relatively small strain using samples do not show necking, the behaviour observed was mainly the fracture process of the connecting chains.

\section{Model Fitting of Internal Fracture in Pre-necking Region}

60 In this section, we further discuss the internal fracture in prenecking region, using some theoretical models. In this process, the $E_{\mathrm{DN}}$ decreases gradually, which means that 1) elastically effective PAMPS chains consist of various lengths, and 2) the fracture of such PAMPS occurs in order of PAMPS chain length; ${ }_{65}$ relatively short chains break at small $\varepsilon$ whereas large ones break at large $\varepsilon$. Thus, the pre-strain dependence of the $E_{\mathrm{DN}}$ (Figure 6) contains information of length distribution of elastically effective PAMPS chains. Wang and Hong have shown that the rate of PAMPS damage for a DN gel that does not show necking follows 70 the log-normal distribution ${ }^{13}$.

$$
\frac{\mathrm{d} \eta}{\mathrm{d} \lambda_{\max }}=-\frac{\eta_{0}}{\sqrt{2 \pi} \lambda_{\max } d} \exp \left[-\frac{1}{2 d^{2}}\left(\ln \frac{\lambda_{\max }-1}{\lambda_{0}-1}\right)^{2}\right]
$$

This derives an error function of the modulus change.

$$
\eta\left(\lambda_{\max }\right)=1-\frac{\eta_{0}}{2}\left[\operatorname{erf}\left(\frac{1}{\sqrt{2} d} \ln \frac{\lambda_{\max }-1}{\lambda_{0}-1}\right)+1\right]
$$

75 where $\eta$ measures the $E_{\mathrm{DN}}$ of pre-stretched $\mathrm{DN}$ gels relative to that of the virgin phase, and is related to the fraction of broken polymer chains in elastically effective PAMPS chains, $\lambda_{\max }$ is the maximum stretching ratio $\left(=\varepsilon_{\max }+1\right)$ that the sample has experienced, $\lambda_{0}$ is approximately the stretching ratio at which the 80 elastically effective chains break most probably, $d$ is a positive parameter controlling the width of the probability distribution, and $\eta_{0}$ is a dimensionless parameter for normalization. The physical meaning of this model is that, the PAMPS chain breaks when it is stretched to the critical strain proportional to its 85 contour length, and the contour length distribution of the PAMPS chains is close to log-normal. Here we adopt the same model to fit our experimental result in Figure 6 to obtain structural information. Figure 9(a) shows the best fitting curve for our experiment in the pre-necking region using eq.(14) and fitting 90 parameters $\eta_{0}=0.89, d=0.50$, and $\lambda_{0}=1.66$. The fitting seems quite well. Based on the model and the obtained fitting parameters, the critical strain distribution at which the elastically effective PAMPS chain breaks has been calculated using eq.(13), as shown in Figure 9(b). As the critical strain at breaking is proportional to 95 the contour length of PAMPS chain, Figure 9(b) also represents the elastically effective PAMPS chain length distribution scaled by an initial length. According to the hierarchical structural model, this means that PAMPS chains connecting the microgels in the intact DN gel have a log normal distribution. Note that this 100 length distribution does not represent that of the elastically 
(a)
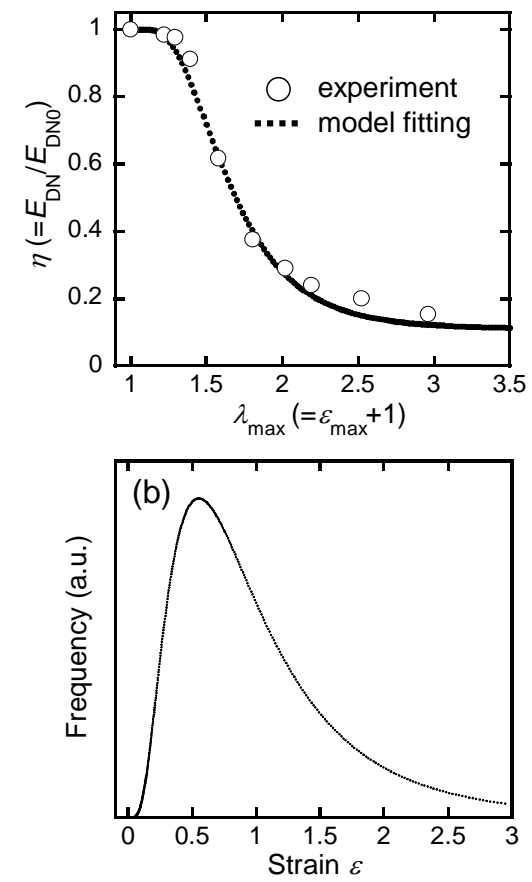

Figure 9 (a) Best fitting curves for the deformation-induced softening using Wang \& Hong's model. (b) Log-normal distribution of critical strain at which the PAMPS chain breaks. Since the critical strain is proportional to the contour length of PAMPS chain, the result also 5 indicates the "elastically effective" PAMPS chain length distribution. These "elastically effective" chains correspond to the PAMPS chains that connect different microgels.

ineffective PAMPS chains in the microgels.

We further discuss the behaviour of the PAMPS chains at large 10 deformation by using Gent hyperelastic model, which is the simple deformation model of rubbers with consideration for finite extensibility and strain hardening of polymer chains ${ }^{10,27}$. We chose this model because of the best fitting in comparison to other hyperelastic models. Gent model predicts uniaxial stress15 strain behaviours as:

$$
\sigma=\frac{E}{3} \times \frac{\left(\lambda_{\mathrm{x}}-\lambda_{\mathrm{x}}^{-2}\right)}{1-\left(\mathrm{J}_{1} / \mathrm{J}_{\mathrm{m}}\right)}
$$

where $E$ is the elastic modulus, $J_{1}$ is the first stress invariant, $\lambda_{\mathrm{x}}$ is the principal stretch ratio in the tensile direction and $J_{\mathrm{m}}$ is an adjustable parameter representing finite extensibility as a 20 maximum allowable value for the first stress invariant. $J_{1}=\lambda_{\mathrm{x}}^{2}+2 \lambda_{\mathrm{x}}^{-1}-3$ and $\lambda_{\mathrm{x}}=\varepsilon+1$ for this system. We fitted the second loading curves of DN gels that experienced various maximum strains $\varepsilon_{\max }$ using Eq. 15 , and only $J_{\mathrm{m}}$ is the fitting parameter. As shown in Figure 10(a), Gent model can fit the experimental 25 results significantly well initially, but less well near $\varepsilon_{\max }$. Through the fitting, we obtained the fitted $J_{\mathrm{m}}$ value for various $\varepsilon_{\max }$. Figure 10(b) shows the relationship between $J_{\mathrm{m}}$ and $J_{1 \max }$, which is the maximum $J_{1}$ that the sample experienced and calculated from $\varepsilon_{\max }$. We found a critical $\varepsilon_{\max }$ of $58 \%$, above which $J_{\mathrm{m}}$ is exactly 30 identical to $J_{1 \max }$, that is, $J_{\mathrm{m}}=J_{1 \max }$. In contrast, at lower $\varepsilon_{\max }$ than $0.58, J_{\mathrm{m}}$ becomes larger than $J_{1 \max }$. The deviation of $J_{\mathrm{m}}$ from $J_{1 \max }$ below a critical $\varepsilon_{\max }$ of $58 \%$ is consistent with the PAMPS chain length distribution that exhibit a maximum frequency at strain
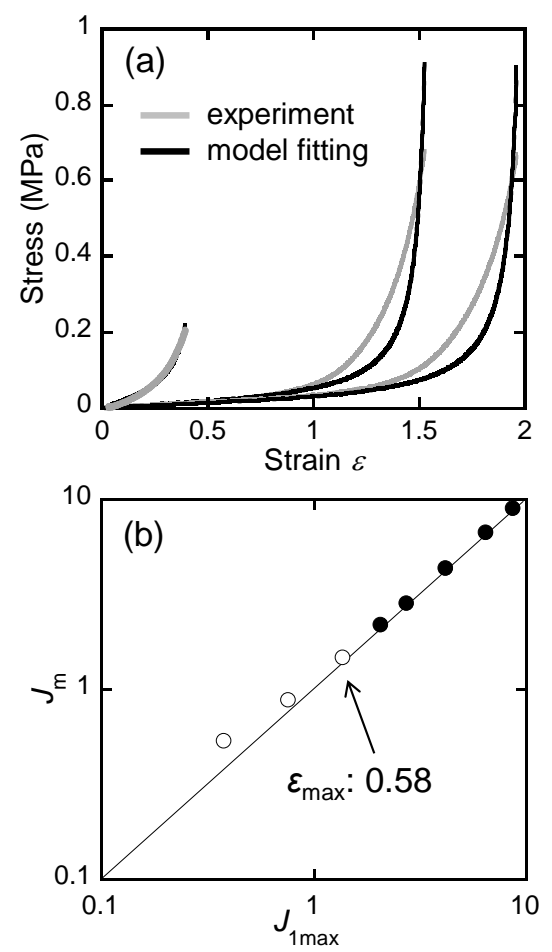

Figure 10 (a) The experimental second loading curves of the DN gels 35 with several prestrain $\varepsilon$, and the theoretical loading curves fitted with

Gent hyperelastic model for them. (b) The relationship between experimental $J_{1 \max }$ and fitted $J_{\mathrm{m}}$. At high $J_{1 \max }$ (black circle) values $J_{\mathrm{m}}$ are almost identical to $J_{1 \max }$ whereas at low $J_{1 \max }$ (white circle) $J_{\mathrm{m}}$ becomes a bit larger than $J_{1 \max }$.

${ }_{40}$ 50\%, as shown in Figure 9(b), although results in Figures 9 and 10 are derived from two independent models. This can be explained as follows: $J_{\mathrm{m}}$ is the maximum allowable stress invariant representing the maximum extensibility of elastically effective polymer chains, determined by chain length. For an ${ }_{45}$ ideal polymer network with homogeneous chain length between cross-linking points, $J_{\mathrm{m}}$ only depends on the length of such polymer chains. In the DN gel system, however, chain length is widely distributed. In this case, which chain length does most affect to $J_{\mathrm{m}}$ ? We can argue from following two points. First, $J_{\mathrm{m}}$ is ${ }_{50}$ strongly affected by the length of elastically effective chains of the largest number (mode value of the length), because degree of contribution to $J_{\mathrm{m}}$ should be simply proportional to number of chains. Second, $J_{\mathrm{m}}$ is also strongly affected by the length of the shortest elastically effective chains, because such chains are in 55 the most stretched state at the fracture point; i.e. they feel the largest stress among all the chains. According to Figure 9(b), the strain that fracture of elastically effective PAMPS mostly occurs (corresponding to the mode of the chain length) is around 50\%. Thus, when the applied $\varepsilon_{\max }$ is lower than $50 \%$, mode value of $\varepsilon$ 60 is larger than the applied $\varepsilon_{\max }$. As a result, $J_{\mathrm{m}}$ becomes slightly larger than $J_{1 \max }$ as the first effect is not negligible. On the other hand, when $\varepsilon_{\max }$ is larger than $50 \%$, as relatively short chain are already broken, length of the shortest remained chains corresponds to the mode value of chain length distribution. As a ${ }_{65}$ result, $J_{\mathrm{m}}$ becomes the same as $J_{1 \max }$. These findings are a little bit different from the previous results, which show that $J_{\mathrm{m}}$ is always smaller than $J_{1 \max }$ although $J_{\mathrm{m}}$ increases proportionally to $J_{1 \max }{ }^{10}$. This is because the previous work only studied in a small $J_{1 \max }$ 
range $\left(J_{1 \max }<4\right)$ in which $J_{\mathrm{m}}<J_{1 \max }$ is still dominant.

\section{Re-Swelling after deformation}

Figure 11 shows the dependence of the re-swelling degree, $q_{\mathrm{w}}$ (g/g, defined in weight change), on $\varepsilon_{\max }$ for the $t$-DN gels. 5 Starting from a value of $10, q_{\mathrm{w}}$ increased monotonically with $\varepsilon_{\mathrm{max}}$. As mentioned in the introduction, this result also represents evidence of the internal fracture of the PAMPS network. It should be noted that the increasing rate of $q_{\mathrm{w}}$ significantly increased in the hardening region, and finally $q_{\mathrm{w}}$ reached a value of 65 (98.5 $10 \mathrm{wt} \%$ of water content). The variation in the slope at the neckinghardening boundary also corresponds to hierarchical internal fracture of DN gels. In the pre-necking and necking regions, the PAMPS network within DN gels remains partially continuous

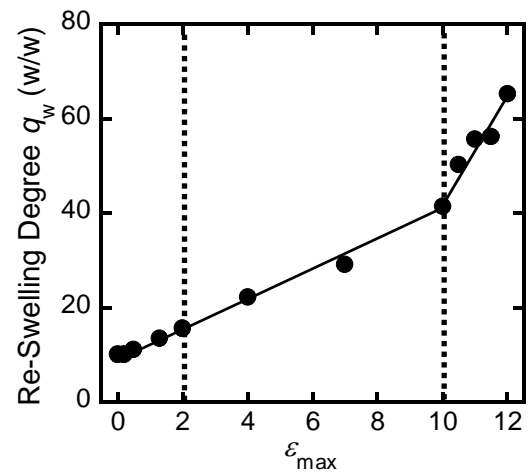

Figure 11 Dependence of the re-swelling degree $q_{\mathrm{w}}$ on $\varepsilon_{\max }$ of the reswollen $t$-DN gels. The solid lines are guides for the eye. Two dotted vertical lines indicate the boundary of the three regions described in Figure 3(a).
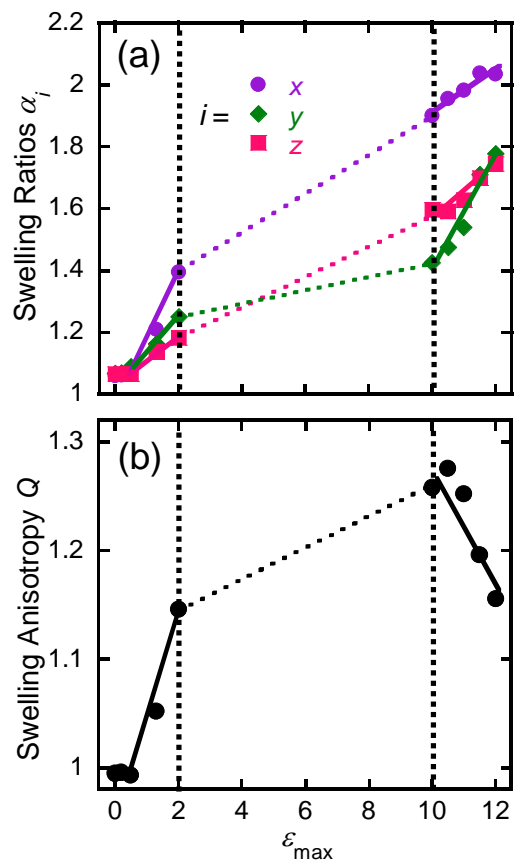

Figure 12 (a) Swelling ratios of the $x, y$, and $z$-axis, $\alpha_{x}, \alpha_{y}$, and $\alpha_{z}$, of reswollen $t$-DN gels pre-stretched at various strains $\varepsilon_{\max }$ along X-axis. (b)

20 Swelling anisotropy, $Q$, calculated from (a). The lines are guides for the eye. Two dotted vertical lines indicate the boundary of the three regions described in Figure 2a. No data was measured in necking region due to inhomogeneous deformation. (a)
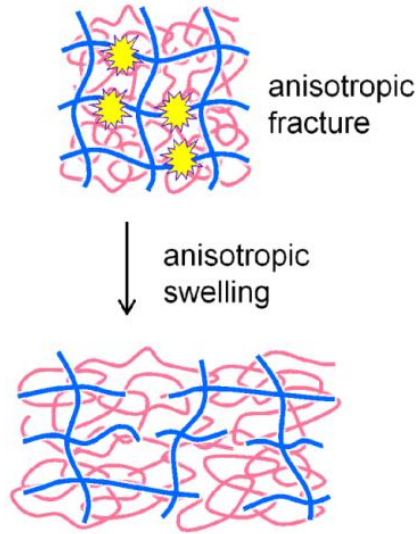

(b)
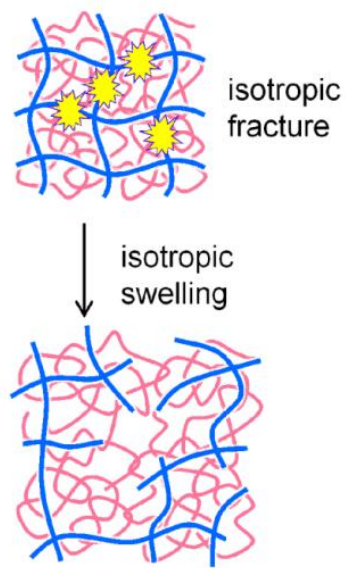

Figure 13 Swelling mechanism of the DN gels after experienced a 25 deformation. (a) Anisotropic fracture of the PAMPS network induces an anisotropic re-swelling. (b) Isotropic fracture induces an isotropic reswelling.

and can still restrain the swelling. In these regions the partial breakage of the PAMPS network cage leads to a certain release of 30 swelling freedom for PAAm network. In contrast, in the hardening region where the PAMPS network fractures into discontinuous fragments, a substantial release of PAAm from the PAMPS network cage is obtained, and the PAMPS fragment may work as a huge cross-linking point of the PAAm network.

35 Figure 12(a) shows the dependence of $\varepsilon_{\max }$ on swelling ratios $\alpha_{x}, \alpha_{y}$, and $\alpha_{z}$ (defined in length change) of the re-swollen $t$-DN gels that were uniaxially elongated along the $x$-axis. In the prenecking region, $\alpha_{y}$ and $\alpha_{z}$ increased modestly whereas $\alpha_{x}$ increased significantly. This observation suggests that the pre40 strained DN gels swelled more in the tensile direction. In contrast, in the hardening region, all the $\alpha$ values seem to increase similarly. We quantify such difference in the degree of anisotropy with the factor $Q$, which indicates the increase in the value of $\alpha_{x}$ compared to the average values of $\alpha_{y}$ and $\alpha_{z}$. Figure 12(b) shows

${ }_{45}$ the value of $Q$ of the re-swollen $t$-DN gels $v s \varepsilon_{\max }$. The value of $Q$ increased with increasing $\varepsilon$ in the pre-necking region (increase of the swelling anisotropy) whereas it decreased with increasing $\varepsilon$ in the hardening region (decrease of the swelling anisotropy).

The increase of $Q$ in the pre-necking region should correspond 50 to the anisotropic internal fracture of the PAMPS network, as schematically illustrated in Figure 13(a). A larger value of $\alpha_{x}$ compared to $\alpha_{y}$ or $\alpha_{z}$ in the pre-necking region indicates that the tension in the PAMPS network against the swelling mainly decreased in the $x$-axis. This implies that the PAMPS chains in

55 the $x$-axis mainly broke during deformation at low $\varepsilon$. This result probably relate to the small-angle neutron scattering results by Tominaga et al., which indicates existence of the periodical structure (size: $\sim 1.5 \mu \mathrm{m}$ ) within PAMPS network of the uniaxially deformed DN gel existing only in tensile direction ${ }^{26}$. In ${ }_{60}$ contrast, a decrease in $Q$ in the hardening region suggests an isotropic fracture, as shown in Figure 13(b). The even increase in $\alpha_{x}, \alpha_{y}$ and $\alpha_{z}$ implies that the PAMPS chains were broken similarly, regardless of their original directions, at high $\varepsilon$. 
(a) pre-necking
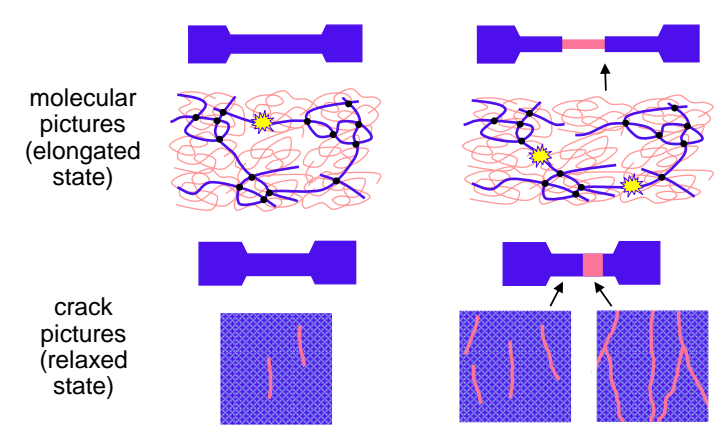

crack

direction

PAMPS

network

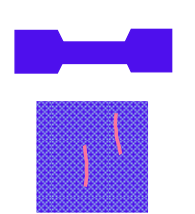

(b) necking

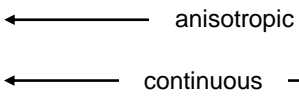

(c) hardening

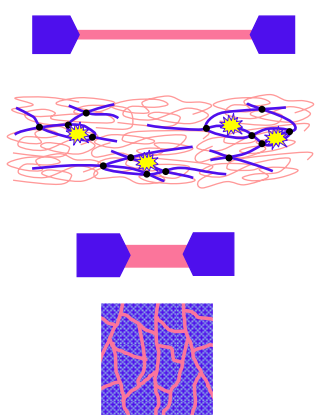

isotropic

Figure 14 Estimated internal fracture process of DN gels in the three regions. The dilute brittle network (blue), PAMPS, has short chains with hierarchical structure consisting of microgels connected by elastically effective chains. The concentrated ductile network (red), PAAm, has very long chains. (a) In the pre-necking region, the elastically effective PAMPS chains which originally directs along the tensile direction preferentially break. The PAMPS network is still marginally continuous macroscopically. (b) In the necking region, two phases co-exist in gels: the continuous phase and discontinuous phase of

5 brittle network, as represented by the isolated red lines and percolated red lines, respectively, in the crack pictures. At the boundary of the two phases, the continuous PAMPS network breaks into discontinuous fragments with anisotropic plate-like shape. (c) In the hardening region, all the polymer chains are oriented along the tensile direction due to large strain, regardless of their initial orientation. As a result, the PAMPS fragments further break into smaller isotropic clusters.

A question remains concerning the origin of the orientation 10 dependence of $Q$. We believe that this dependency can be explained by considering orientation of the PAMPS chains. The fundamental assumption is that the PAMPS chains oriented in the tensile direction ( $x$-axis) mainly break because stress is concentrated within those chains during uniaxial deformation. 15 Distribution of the direction of the PAMPS chains strongly depends on strain; the chain orientation should be random in the relaxed state whereas the chains may be oriented in the stretched state. We estimated degree of the PAMPS chain orientation in DN gels under uniaxial stretching quantitatively based on the 20 simple model. The model and detailed results are shown in Figure S2 of Supplemental Information. Briefly, in the pre-necking region $(\varepsilon<2)$ the degree of chain orientation after deformation was still in the order of initial chain orientation. This implies that the PAMPS chains which originally orient in the tensile direction 25 breaks preferentially in this region. In the hardening region, however, virtually all chains are completely oriented along the tensile direction, regardless their initial orientation. This implies that the fracture of the PAMPS chains occurs evenly for all the chains regardless of their original orientations in this region.

30

\section{A Summary for Internal Fracture Process}

Summarizing the results and the discussion, we propose the following internal fracture process for uniaxial elongation of DN gels. The brittle network, PAMPS, has a hierarchical structure 35 consisting of microgels connected by elastically effective chains. In the pre-necking region, among the elastically effective PAMPS chains, the chains "originally" orient along the $x$-axis mainly break as shown in Figure 14(a). This argument is justified by two experimental observations: one is the increase of the swelling 40 anisotropy $Q$, and the other is the obvious decrease of the modulus $E_{\mathrm{DN}}$. The former implies that the PAMPS chains originally orient along the $x$-axis break selectively, and the latter implies that the fractured chains are elastically effective. The PAMPS network remains marginally continuous in the pre${ }_{45}$ necking region despite such internal fracture, because $E_{\mathrm{DN}}$ remains slightly larger than its convergent value, which implies that some elastically effective PAMPS chains are still exist in the gels. The elastically effective PAMPS chains break in order of length, corresponding to gradual decrease of $E_{\mathrm{DN}}$. In the necking 50 region, the relatively hard region and the soft region co-exist. At the boundary, the PAMPS network breaks into anisotropic fragments and becomes discontinuous as shown in Figure 14(b). This argument is justified by the behaviours of $E_{\mathrm{DN}}$ and a large $Q$. The decrease in $E_{\mathrm{DN}}$ stops at the end of the necking region and 55 becomes almost constant thereafter. This suggests that the elastically effective PAMPS chains of the bulk sample have completely fractured into discontinuous fragments. As $Q$ remains large at the end of the necking region, the PAMPS chains originally directed along the $x$-axis still mainly break. Therefore, 60 we assume that the shape of PAMPS fragments is not isotropic but has a rectangular or plate-like feature. Our group observed a wrinkled structure in ultra-thin DN gels after tensile or tearing tests ${ }^{11}$. The orientation of the wrinkles was always perpendicular to the tensile direction. This is well in agreement with our present 65 argument. In the hardening region, all the polymer chains are oriented along the tensile direction due to large strain, regardless of their initial orientation. As a result, PAMPS chains in the PAMPS fragments further break into smaller isotropic clusters, as shown in Figure 14(c). The evidence of this argument is the ${ }_{70}$ decrease of the swelling anisotropy $Q$. In this region, DN gels show strain hardening. This implies that all the polymer chains in DN gels are oriented along the $x$-axis due to the very large elongation. Thus, the fracture of PAMPS chains occurs evenly with no relation to their original direction. As shown in Figure 7515 (c), chains originally directed perpendicular to the $x$-axis are oriented in the tensile direction and break. As a result, the fractured PAMPS fragments show an isotropic shape in the 
relaxed state.

It should be noted that although the hierarchical structure of the brittle network play the decisive role in the internal fracture processes of the DN gel, it is still an open question whether the 5 hierarchical structure, or the heterogeneity, of the brittle network is crucial for the efficient internal fracture of the DN gels. Study addressing this issue is under progress.

\section{Acknowledgements}

This research was financially supported by a Grant-in-Aid for 10 Scientific Research (S) (No. 124225006) from Japan Society for the Promotion of Science (JSPS).

\section{References}

1 A. H. Cottrell and B. A. Bilby, Proc. Phys. Soc. A, 1949, 62, 49.

2 N. W. Brooks, A. P. Unwin, R. A. Duckett and I. M. Ward, J. 15 Macromol. Sci. Phys., 1995, 34, 29.

3 R. P. Kambour, Polymer, 1964, 5, 143.; H. R. Brown and I. M. Ward, Polymer, 1973, 14, 469.

4 G. J. Lake and A. G. Thomas, Proc. R. Soc. London, Ser. A, 1967, 300, 108 .

205 S. Naficy, H. R. Brown, J. M. Razal, G. M. Spinks and P. G. Whitten, Aust. J. Chem., 2011, 64, 1007.; K. Haraguchi, Polym. J., 2011, 43, 223.

6 J. P. Gong, Y. Katsuyama, T. Kurokawa and Y. Osada, Adv. Mater., 2003, 15, 1155.

257 T. Nakajima, H. Furukawa, Y. Tanaka, T. Kurokawa, Y. Osada and J. P. Gong, Macromolecules, 2009, 42, 2184.

8 Y. Tanaka, R. Kuwabara, Y.-H. Na, T. Kurokawa, J. P. Gong and Y. Osada, J. Phys. Chem. B, 2005, 109, 11559. ; J. P. Gong, Soft Matter, 2010, 6, 2583.

309 Y.-H. Na, Y. Tanaka, Y. Kawauchi, H. Furukawa, T. Sumiyoshi, J. P. Gong and Y. Osada, Macromolecules, 2006, 39, 4641.; Y. Tanaka, Y. Kawauchi, T. Kurokawa, H. Furukawa, T. Okajima and J. P. Gong, Macromol. Rapid Commun., 2008, 29, 1514.

10 R. Webber, C. Creton, H. R. Brown and J. P. Gong, Macromolecules, 2007, 40, 2917.

11 S. Liang, Z. L. Wu, J. Hu, T. Kurokawa, Q. M. Yu and J. P. Gong, Macromolecules, 2011, 44, 3016.

12 H. R. Brown, Macromolecules 2007, 40, 3815.; Y. Tanaka, Europhys. Lett., 2007, 78, 56005

4013 X. Wang and W. Hong, Soft Matter, 2011, 7, 8576.

14 X. Zhao, J. Mech. Phys. Solids, 2012, 60, 319.

15 P. J. Flory and J. Rehner, J. Chem. Phys., 1943, 11, 521.

16 T. Nakajima, H. Furukawa, J. P. Gong, E. K. Lin and W.-L. Wu, Macromol. Symp., 2010, 291-292, 122.

4517 H. Itagaki, T. Kurokawa, H. Furukawa, T. Nakajima, Y. Katsumoto and J. P. Gong, Macromolecules, 2010, 43, 9495.

18 J. Diani, B. Fayolle and P. Gilormini, Eur. Polym. J., 2009, 45, 601.; A. D. Drozdov, Mech. Res. Commun., 2009, 36, 437.; A. Dorfmann and R. W. Ogden, Int. J. Solids. Struct., 2004, 41, 1855.

5019 M. Rubinstein, R. H. Colby, A. V. Dobrynin, J. F. Joanny, Macromolecules 1996, 29, 398.

20 M. A. Haque, T. Kurokawa, G. Kamita, Y. Yue and J. P. Gong, Chem. Mater., 2011, 23, 5200.

21 A. A. Zavitsas, J. Phys. Chem., 1987, 91, 5573.

5522 T. Tominaga, V. R. Tirumala, E. K. Lin, J. P. Gong, H. Furukawa, Y. Osada and W.-L. Wu, Polymer, 2007, 48, 7449.

23 J. Bastide and L. Leibler, Macromolecules, 1988, 21, 2647.; M. Shibayama, Macromol. Chem. Phys., 1998, 199, 1.

24 T. Norisuye, N. Masui, Y. Kida, D. Ikuta, E. Kokufuta, S. Ito, S.

60 Panyukov, and M. Shibayama, Polymer, 2002, 43, 5289.; M. Y. Kizilay and O. Okay, Macromolecules, 2003, 36, 6856.

25 M. Huang, H. Furukawa, Y. Tanaka, T. Nakajima, Y. Osada and J. P. Gong, Macromolecules, 2007, 40, 6658.
26 Y.-H. Na, Y. Tanaka, Y. Kawauchi, H. Furukawa, T. Sumiyoshi, J. P. 65 Gong and Y. Osada, Macromolecules, 2006, 39, 4641.

27 A. N. Gent, Rubber Chem. Technol., 1996, 69, 59.

${ }^{a}$ Faculty of Advanced Life Science, Hokkaido University, N10W8, Kita-ku, 70 Sapporo, Japan. Fax: +81-11-706-2774; Tel: +81-11-706-2774; E-mail: gong@sci.hokudai.ac.jp

${ }^{b}$ Creative Research Institution, Hokkaido University, N21W15, Kita-ku, Sapporo, Japan.

${ }^{c}$ Graduate School of Life Science, Hokkaido University, N10W8, Kita-ku, 75 Sapporo, Japan.

${ }^{d}$ National Institute of Standards and Technology, 100 Bureau Drive, Gaithersburg, MD, USA 


\section{Table of contents entry}

We have quantificaly analysed hierarchical and anisotropic internal fracture process of tough double network (DN) gels with tensile 5 mechanical hysteresis and re-swelling measurements.

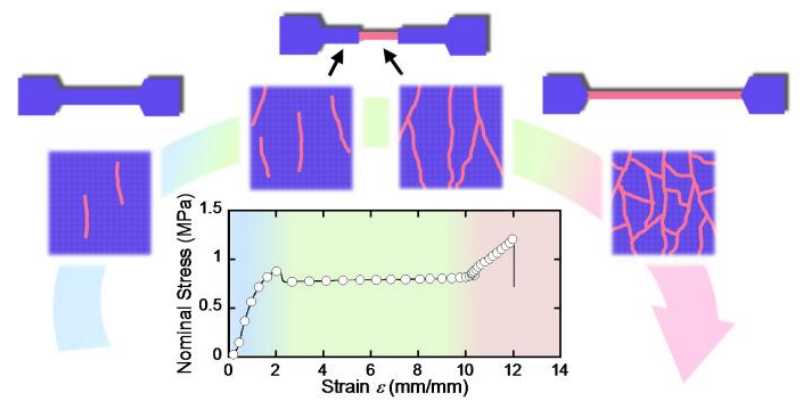

